\title{
Quantitative organization of the excitatory synapses of the primate cerebellar nuclei: further evidence for a specialized architecture underlying the primate cerebellum
}

\author{
Haian Mao ${ }^{1} \cdot$ Salah Hamodeh ${ }^{1} \cdot$ Angelos Skodras $^{2} \cdot$ Fahad Sultan $^{3}$ \\ Received: 21 September 2018 / Accepted: 4 May 2019 / Published online: 17 May 2019 \\ (c) The Author(s) 2019
}

\begin{abstract}
The cerebellar intrinsic connectivity is of remarkable regularity with a similar build repeated many times over. However, several modifications of this basic circuitry occur that can provide important clues to evolutionary adaptations. We have observed differences in the wiring of the cerebellar output structures (the deep cerebellar nuclei, DCN) with higher dendritic length density in the phylogenetically newer DCN. In rats, we showed that an increase in wiring is associated with an increase in the presynaptic vesicular glutamate transporter 1 (vGluT1). In this study, we have extended our analysis to the rhesus monkey and can show similarities and differences between the two species. The similarities confirm a higher density in vGluT1+ boutons in the lateral (LN/dentate) and posterior interpositus nucleus compared to the phylogenetically older DCN. In general, we also observe a lower density of vGluT1 and 2+ boutons in the monkey, which however, yields a similar number of excitatory boutons per neuron in both species. The only exception is the vGlut1+ boutons in the macaque LN/ dentate, which showed a significantly lower number of vGluT1+ boutons per neuron. We also detected a higher percentage of co-labelled vGluT1 and 2 boutons in the macaque than we found in the rat. In summary, these results confirm that the hyposcalled dendrites of the monkey LN/dentate also show a lower number of vGluT1+boutons per neuron. These results provide further support of our model relating the dendritic morphology of the LN/dentate neurons to the morphology of the specially enlarged LN/dentate nucleus in primates.
\end{abstract}

Keywords Vesicular glutamate transporter · Lipofuscin fluorescence removal · Deep cerebellar nuclei · Quantitative immunofluorescence $\cdot$ Comparative neuroanatomy

Electronic supplementary material The online version of this article (https://doi.org/10.1007/s00429-019-01888-8) contains supplementary material, which is available to authorized users.

Fahad Sultan

fahad.sultan@umu.se

1 Department of Cognitive Neurology, Hertie Institute for Clinical Brain Research, Otfried-Müller-Str. 27, 72076 Tübingen, Germany

2 Department of Cellular Neurology, Hertie Institute for Clinical Brain Research, Otfried-Müller-Str. 27, 72076 Tübingen, Germany

3 Department of Integrative Medical Biology, Umeå University, Linnéus Väg 9, 90187 Umeå, Sweden

\section{Introduction}

The deep cerebellar nuclei (DCN) are a key component of the cerebellar circuitry and the main output of the cerebellum. The activity of DCN neurons is controlled by the integration of inhibitory synaptic input from the cerebellar cortex (i.e., the Purkinje cell axons) and excitatory input from the precerebellar nuclei. The precerebellar afferents are from a wide source of brainstem nuclei conveying vestibular, somatosensory, visual, auditory stimuli and from the basilar pontine nuclei conveying cerebral information. There are two large groups of output neurons within the DCN. An excitatory projection group that provides glutamatergic inputs to different brain regions within the brainstem (e.g., the red nucleus) and the cerebral cortex (via specific thalamic relay nuclei). In contrast, the other group is GABAergic and only targets the inferior olive nuclei. Both paths are also involved in recurrent circuits that feed back 
to the DCN and cerebellar cortex either via the collaterals of the mossy fibers or of the climbing fibers. In classical conditioning learning tasks, such as the Pavlovian eyeblink conditioning, the mossy fibers have been shown to convey the conditioned stimulus, whereas the climbing fibers provide the unconditioned stimulus to the DCN and cerebellar cortex (Boele et al. 2013; Lee et al. 2015). This dichotomy is further underlined by the presence of two vesicular glutamate transporters (vGluT1 and 2) used by the two paths in different ways; the climbing fibers exclusively use vGluT2, while all mossy fiber express vGluT1. It is noteworthy that a substantial subpopulation of mossy fibers also co-expresses vGluT2 (Hisano et al. 2002; Hioki et al. 2003; Boulland et al. 2004) and it is still unclear what functional role this co-labelling provides.

Based on the expression of vGluT1 and 2 in the climbing and mossy fiber DCN collaterals, immunolabeling these two transporters is an ideal way of marking all the excitatory axon terminals in the DCN. Using this approach together with systematic unbiased sampling of the rat DCN we were able to show that both transporters are expressed in the DCN in similar proportions. However, we also found that vGluT1+ bouton density is significantly higher in the phylogenetically newer lateral nucleus/dentate (LN/dentate) and posterior interpositus nucleus (PIN) compared to the phylogenetically older DCN (medial nucleus: $\mathrm{MN}$ and anterior interpositus nucleus: AIN). In contrast the vGluT2+bouton density did not show any significant difference between the different DCN (Mao et al. 2018). These findings is in agreement with the general brain pattern of vGluT1 and 2 expression, with the former predominantly expressed in the phylogenetically newer brain regions (cerebral cortex) and the later in subcortical regions such as the midbrain and thalamic nuclei (Fremeau et al. 2004). Based on this view our first prediction would be that in primates with an enlarged neocerebellum the density of vGluT1+ boutons in the $\mathrm{LN} /$ dentate should also be increased within this nucleus. However, it is not clear whether the results from the rat LN/ dentate can be generalized to primates. In fact a different prediction emerges if we look at the wiring components within the DCN. In a previous study (Hamodeh et al. 2017), we quantified and compared the wiring components between the rat and rhesus monkey DCN. We found that although the dendritic length density (dendritic length per tissue volume) was increased within the $\mathrm{LN} /$ dentate, a different picture emerged when we compared the dendritic length per neuron (dendritic length density normalized by neuron density). Here we found that the LN/dentate had less dendritic length per neuron than would have been predicted by the overall volume of the primate $\mathrm{LN} /$ dentate. We termed this observation hyposcaling of dendrites and related it to the flattened shape of the LN/dentate. Therefore, the quantification of the vGluTs in the primate LN/dentate could clarify whether we also observe a hyposcaling of the excitatory synapses in this nucleus.

Our immunofluorescence approach that we established in the rodent should also be applicable to the primate brain tissue on the basis of antigen homology (Garcia-Marin et al. 2013). However, the presence of lipofuscin complicates this approach (Terman and Brunk 2004), due to its broad emission spectrum. In this study, we, therefore, modified an approach from flow cytometry (Steinkamp and Stewart 1986; Roederer and Murphy 1986; Billinton and Knight 2001) to remove the lipofuscin emission. This required acquiring an additional lipofuscin specific channel and using that to mask the vGluT1 and vGluT2 signals during postprocessing.

\section{Experimental procedure}

\section{Tissue preparation}

All animal experiments were carried out in accordance with the Society for Neuroscience and local German authorities (approved by the regional authorities (Regierungspräsidium Tübingen). Brain tissue from two male adult macaques was used in this study (D98: body weight $17 \mathrm{~kg}$ and 18 years old, H01: body weight $9 \mathrm{~kg}$ and 13 years old). Following premedication with a mixture of ketamine $(20 \mathrm{mg} / 100 \mathrm{~g}$ body weight), xylazine ( $2 \mathrm{mg} / 100 \mathrm{~g}$ body weight) deep anesthesia was induced with pentobarbital $(400 \mathrm{mg} / \mathrm{kg})$. The animals were then perfused transcardially by $0.1 \mathrm{M} \mathrm{PB}$ and then by $4 \%$ ice-cold paraformaldehyde in $0.1 \mathrm{M}$ phosphate buffer (PB) at $\mathrm{pH}$ 7.4. The brain was immediately dissected out of the skull and then cryoprotected in an ascending concentration of sucrose $(10 \%, 20 \%$, and $30 \%$ in $0.1 \mathrm{M} \mathrm{PB})$. The cerebellum was removed from the brainstem and mounted on a microtome with the lateral side facing the freezing platform. Sections of $40 \mu \mathrm{m}$ (D98) or $50 \mu \mathrm{m}$ (H01) were serially acquired and stored in $0.1 \mathrm{M}$ PB. Immunofluorescence staining was carried out on free floating sections. Prior to primary antibody incubation, sections were washed three times in $0.1 \mathrm{M} \mathrm{PB}$ for 5 min each time and then blocked in 0.1 M PB with $10 \%$ horse serum (PAA Laboratories, Coelbe, Germany) and $0.3 \%$ Triton X-100 at room temperature for $1 \mathrm{~h}$.

\section{Immunofluorescence}

The concentration and incubation times were tested for each antibody in single stains. The optimized conditions were then used for the multiple stains. We performed a quadruple staining protocol for vGluT1, vGluT2, Purkinje Cell Protein 2(PCP2), and potassium channel KCNC3/Kv3.3 on $\mathrm{H} 01$, and a further quadruple staining protocol for vGluT1, 
vGluT2, MAP2 and KCNC3/kv3.3 on D98. Data of the Kv3.3 were used for a subsequent publication. The sections were first incubated with goat vGluT1 (sc-13320, Santa Cruz Biotechnology, Texas) at $1: 1000$ and $4{ }^{\circ} \mathrm{C}$ for $16 \mathrm{~h}$, followed by $3 \times$ washes in $0.1 \mathrm{M} \mathrm{PB}$ and then incubated with donkey anti goat Alexa Fluor 488 at 1:500 for $2 \mathrm{~h}$ at room temperature. The sections were then washed three times in PB before incubation with a combination of three antibodies of vGluT2 (1:1000, lot number: 135404, Synaptic systems, North Saanich British Columbia), Kv3.3 (1:500, lot number: APC-102, Alomone labs, Israel) and PCP2 (1:200, lot number: sc-137064, Santa Cruz Biotechnology, Texas) or MAP2 (1:200, lot number: M1406, Sigma Aldrich) at $4{ }^{\circ} \mathrm{C}$ for $16 \mathrm{~h}$. The sections were then washed and incubated with the combinations of the three secondary antibodies: goat anti-guinea pig Alexa Fluor 633 (1:500, A21105, Invitrogen, California), goat anti-rabbit Cy3 (1:500, lot number: 81-6115, Invitrogen, California) and goat anti-mouse Alexa Fluor 405 (1:500, lot number: A31553, Invitrogen, California) for $2 \mathrm{~h}$ at room temperature. Sections were then washed and mounted in glycerol on glass slides with Mowiol 4-88 (Merck, Darmstadt, Germany). The slides were stored at $4{ }^{\circ} \mathrm{C}$.

\section{Preabsorption test of vGluT2 antibody}

To test for vGluT2 antigen-antibody specificity, we mixed $1 \mu \mathrm{l}$ vGluT2 antibody with $2 \mu \mathrm{l}$ of the peptide that was used for immunization and was provided by the supplier. This consisted of Strep-Tag fusion protein (amino acids 510-582 of the rat vGluT2) in $500 \mu \mathrm{l} 0.5 \mathrm{M}$ PB solution with $2 \%$ horse serum and $0.1 \%$ tritonX-100. The mixture was rotated at room temperature for $1 \mathrm{~h}$ to allow for full interaction of the peptide with the antibody. As a control solution we used $1 \mu \mathrm{l}$ vGluT2 antibody diluted in $500 \mu 10.5 \mathrm{M}$ PB solution with $2 \%$ horse serum and $0.1 \%$ tritonX-100. Two adjacent cerebellar sections from the same series of monkey H01 were washed and blocked. One section was incubated in the antigen-antibody mixture and the other in the control solution for $48 \mathrm{~h}$ at $4^{\circ}$ with constant rotation. The two sections were washed and incubated with goat anti-guinea pig Alexa Flour 633 1:1000 for $2 \mathrm{~h}$ at room temperature. The sections were washed, mounted, and scanned with laser confocal microscope LSM 510. VGluT2 channel was scanned under the excitation of $633 \mathrm{~nm}$ and emission at $650-750 \mathrm{~nm}$, while lipofuscin channel was scanned under the excitation of $405 \mathrm{~nm}$ and emission at $650-750 \mathrm{~nm}$.

\section{Data acquisition}

Images were acquired on a laser scanning confocal microscope (LSM 510, Carl Zeiss, Jena, Germany). We took overview images of vGluT1 staining for every section with $488 \mathrm{~nm}$ excitation and emission band pass filtering $505-550 \mathrm{~nm}$ at low magnification $(10 \times$ objective $)$. Probe positions were determined by first marking an identifiable origin $(x, y$ coordinates: 0,0$)$ and then determining the position of the other probes in the DCN region at a regular spacing of $1000 \mu \mathrm{m} \times 1000 \mu \mathrm{m}$. The location of the origin point was chosen from an easily identifiable structure (i.e., vessels) within a core region of the DCN. However, this location differs from slice to slice and is not related to the structures to be analyzed. A $z$-stack was acquired using a $63 \times$ (NA1.4, Oil immersion) objective, applying a $2 \times$ zoom for each probe. The pinhole was set to one airy unit and subsequently optimized for every detection channel to achieve an equal optical slice thickness on all channels. $X Y$ voxel size was set to $0.14 \mu \mathrm{m} \times 0.14 \mu \mathrm{m}$; image digital resolution was set to $512 \times 512$ pixels. The $z$-stack step size was $0.32 \mu \mathrm{m}$ and we took an average of 30 optical sections. We stained and analyzed 13 cerebellar sections (5 slices from D98 and 8 slices from H01) and 168 probes in total were sampled ( 90 from D98, 78 from H01). The fluorescence emitted by lipofuscin granules were recorded with excitation wavelength at $405 \mathrm{~nm}$ and emission wavelength at $650-750 \mathrm{~nm}$. Details of antibodies, excitation wavelength and emission filters are listed in Table 1.

Table 1 Multichannel laser confocal microscopy parameters

\begin{tabular}{lllc}
\hline 1st antibody & 2nd antibody & Excitation (nm) & $\begin{array}{c}\text { Emission (nm) } \\
\text { Track } \\
\text { number }\end{array}$ \\
\hline Goat vGluT1 & Donkey anti-goat Alexa flour 488 & 488 & $505-550$ \\
Guinea pig vGluT2 & Goat anti-guinea pig Alexa flour 633 & 633 & $650-750$ \\
Mouse PCP2 or mouse MAP2 & Goat anti-mouse Alexa flour 405 & 405 & $420-480$ \\
Rabbit kv3.3 & Goat anti rabbit cy3 & 543 & $560-615$ \\
None & None & 405 & $650-750$ \\
\hline
\end{tabular}

Four different secondary antibodies were used with the corresponding primary antibodies. The fluorophores were excited at their optimal wavelength and the emissions were collected using different band pass or long pass filters. Lipofuscin was excited such as to obtain maximal difference between excitation and emission wave length, thereby dissociating it from the other fluorophores. Three tracks were used during the acquisition process to minimize channel interference 
We also determined tissue shrinkage in section thickness by taking four random positions within DCN region for each slice and determining the upper and lower section borders by the staining signal for vGluT1 at a magnification of $20 \mathrm{x}$ (NA0.8, dry). Fifty-two probes were taken from 13 sections. The mean thickness of the slices after mounting was $24.6 \mu \mathrm{m}$ for D98 and $26 \mu \mathrm{m}$ for $\mathrm{H} 01$, while the original sectioning thickness was $40 \mu \mathrm{m}$ and $50 \mu \mathrm{m}$, respectively. Classification of the macaca DCN was based on the description in an earlier paper (Hamodeh et al. 2014).

\section{Data preprocessing and analysis}

Data were acquired as 8-bit format and saved in Zeiss lsm format. Stacks were then deconvoluted using the iterative $(n=10)$ "blind deconvolution" of AutoQuant X3 (Media Cybernetics, Bethesda, MD) with the maximum likelihood estimation and constrained iteration. Following deconvolution, data were saved in Autoquant to 8-bit lsm format.

\section{Lipofuscin masking and extraction of vGluTs boutons}

Lipofuscin has a broader excitation and emission spectrum than commercial fluorophores which are designed with narrow spectra. We acquired the signals from our own fluorophores by regular microscopic settings while simultaneously recording lipofuscin fluorescence signal in an additional channel and subtracted the lipofuscin fluorescence during post-processing.

We performed surface reconstruction to get boundaries of lipofuscin granules using the Imaris software (Bitplane). The lipofuscin surfaces were smoothed at $0.279 \mu \mathrm{m}$ and the background was subtracted using the Ridler and Calvard (R-C) algorithm (Ridler and Calvard 1978) to determine the best thresholding level for this channel. The thresholding level was about $35 / 255$. The reconstructed lipofuscin surfaces were subsequently used to mask the vGluT1 and vGluT2 channel. The voxels inside the lipofuscin mask were set to 0 , while the voxels outside the lipofuscin mask remained unchanged. The background of the masked vGluT1 channel was subtracted. The surface bounding of vGluT1 boutons was smoothed at $0.15 \mu \mathrm{m}$ and the diameter of the largest sphere fitting into the objects was taken at $0.5 \mu \mathrm{m}$. The masked vGluT2 channel was similarly surface-rendered, except that we fitted a Gaussian filter $(\mathrm{SD}=0.3 \mu \mathrm{m})$ beforehand and used a user-defined fixed threshold of 68/255. The threshold for masked vGluT1 channel was determined by the Ridler and Calvard (R-C) algorithm and the resulting mean threshold was 20.1/255 for D98 and 26.2/255 for H01. We used Gaussian filter and user-defined threshold for the masked vGluT2 channel to effectively exclude the background and DCN neuron somata (Mao et al. 2018). The surface-reconstructed boutons were further filtered according to size by excluding surfaces with less than ten voxels (Haass-Koffler et al. 2012).

\section{Shrinkage and density calculation}

The number of surface reconstructed boutons for each probe was exported and normalized to the corrected volume of probe in question. First, we obtained the (uncorrected) section thickness by adding the average diameter of the contours, $1.12 \mu \mathrm{m}$ for vGluT1 and $1.19 \mu \mathrm{m}$ for vGluT2 to account for boutons being only partly contained within a section (Braitenberg and Schüz 1998). The uncorrected probe volume was then obtained by multiplying the probe length $(71.4 \mu \mathrm{m})$ and the probe width $(71.4 \mu \mathrm{m})$ and the corrected depth (varying from probe to probe). Tissue shrinkage affected the thickness of the section and was, therefore, taken into consideration. To determine the extent of the shrinkage, four random positions were chosen within the DCN region for each stain section. All the values were then averaged and the cerebellar sections from D98 were shrunk to $24.6 \mu \mathrm{m}$ thickness, while those of $\mathrm{H} 01$ were shrunk to a thickness of $26 \mu \mathrm{m}$, the original sectioning thicknesses being $40 \mu \mathrm{m}$ for D98 and $50 \mu \mathrm{m}$ for H01. Finally, we divided the uncorrected probe volume with 24.6/40 for D98 and 26/50 for H01 to obtain the corrected probe volumes.

\section{Calculating the co-labelling of vGluT1 and vGluT2 boutons}

The quantification of co-labelling of vGluT1 and 2 was performed by obtaining the intersection of the surface boundaries of the 2 vGluT channels (vGluT1n2). However, we could only perform channel subtractions in Imaris. We, therefore, subtracted vGluT1 from the vGluT1-only channel. The vGluT1-only channel was obtained for each probe by subtracting the vGluT2 channel from the original vGluT1 channel for the rats and from the lipofuscin cleaned vGluT1 channel in the case of the monkey. The number of the colabelled boutons was divided by the vGluT1 bouton number or the vGluT2 bouton number to ascertain the co-labelled percentage for vGluT1 boutons or vGluT2 boutons. The percentages were compared between different nuclei and between the two species.

\section{Statistical analysis}

The vGluT1 and 2 density and volume values were checked for normal distribution and variance homogeneity. Quantile-quantile plots (qqnorm in R, http://www.r-project.org) and Shapiro-tests (package lme4 in R) indicated normal distribution for vGluT1 and vGluT2 volume. Levene's tests (package Rcmdr in R) indicated equal variance for vGluT1 
and vGluT2 volume. The vGluT1 and vGluT2 density values were power transformed by transformTukey (package rcompanion in $\mathrm{R}$ ) to secure normal distribution and equal variance. Since multiple data points were obtained in each subject (Aarts et al. 2014), a linear mixed effect model was used to accommodate the dependence of the data. The linear mixed effects model via restricted maximum likelihood estimations were performed using lme4 package in R. The response was the transformed density or non-transformed volume (for both vGluT1 and 2). The nuclei variance was modelled as a fixed effect, while subject variance was modelled as a random effect in the model. We also constructed a null model with only the random effect. We then compared the two models using a likelihood ratio test to ascertain whether these two models differed significantly from each other. Post hoc multiple comparisons (Tukey contrast) with Holm-Bonferroni correction were performed. The significance level $\alpha$ was set to 0.05 . We also plotted the data with intensity color-coded scatter plots using matlab scripts (Eilers and Goeman 2004).

\section{Results}

\section{vGluT1+ and vGluT2+ staining patterns in the cerebellum}

The vGluT2 immunofluorescence showed a puncta pattern organized in a linear beaded fashion in the molecular layer (ML) and glomeruli-like pattern in the granule cells layer (GCL) of the cerebellar cortex (small arrows in Fig. S1a). There are also granular structures in the Purkinje cells layer (PCL) as described for lipofuscin in the literature [big arrow in Fig. S1a, (Brizzee et al. 1974)]. Not surprisingly, these structures are obtained again under our setting which is used for lipofuscin fluorescence (big arrow, Fig. S1b). The staining pattern of vGluT2 after lipofuscin channel subtraction is consistent with our previous study in the rat using the same antibody (Fig. S1c) (Mao et al. 2018). Preabsorption of vGluT2 antibody with its corresponding peptide abolished the vGluT2 signals in the cortex, pointing to the specificity of the vGluT2 antibody (Fig. S1d, e, f). Similarly, the vGluT2 antibody is also specific in the DCN region (Fig. $\mathrm{S} 1 \mathrm{~g}-1)$. We also controlled the multiple staining patterns in the cerebellar cortex (molecular layer) and DCN of the monkey. As anticipated, parallel fibers were stained by vGluT1 only, whereas climbing fiber presynapses were stained by vGluT2 only (Fig. 1a, b). The vGluT1 and vGluT2 were separated from each other in the molecular layer, as the negative Pearson's coefficient indicates (Fig. 1c). We also compared the staining of vGluT1 and 2 with the PCP2 staining in the DCN and found them to be segregated as in the molecular layer (Fig. 1d, e). However, unlike in the cortex, the vGluT1 and vGluT2 show some colocalization (Pearson's coefficient 0.47). These co-labelled boutons may come from mossy fibers that express both vGluT1 and vGluT2 (Boulland et al. 2004). A comparison of labelling intensity within a voxel confirmed the different patterns; in contrast to the DCN there was no above-threshold intensity co-labelling of vGluT1 and 2 in the molecular layer (Fig. 1c, f). The spatial relationship of the vGluT1 and 2 labelled puncta with their postsynaptic partner, the dendrites from DCN neurons, was also examined in a quadruple staining environment. Most of the vGluT1 and 2 labelled puncta can be observed around the dendrites (Fig. 1g, h). Again, the colocalization of vGluT1 and vGluT2 in the DCN can be seen in this example (Fig. 1i).

\section{Comparison of vGluT1+ and vGluT2+ boutons within DCN}

To obtain the quantitative data of vGluT1+and vGluT2+ bouton density and compare between nuclei, the lipofuscin signal was first detected and eliminated from the vGluT1 and vGluT2 channels. First, we verified the lipofuscin excitation and emission characteristics in unstained macaque cerebellar sections (see supplementary material and Fig. S2). Then we validated our lipofuscin masking approach in the vGluT1 and vGluT2 channels and this is summarized in the supplementary material (see also Fig S2-4).

The structures that are labelled by vGluT1, vGluT2 or both are shown in different nuclei (Fig. 2). Double-labelled structures can be found in all the different nuclei. However, the vGluT1+ boutons are sparser in MN and AIN than in LN and PIN. Nevertheless, the vGluT2+ boutons are more similar between nuclei (Fig. 2a, c, e, g). The surface analysis allowed for a detailed visualization of vGluT1 and vGluT2labelled structures and quantification of the number and volume of the boutons as well as the co-labelled boutons (Fig. 2b, d, f, h).

\section{vGluT1+ and vGluT2+ bouton density and volume within DCN}

We quantified the vGluTs bouton density in the different DCN. The vGluT1+ bouton density is higher in the PIN and $\mathrm{LN}$, with $1.97 \times 10^{6}\left(\mathrm{SEM}=1.78 \times 10^{5}\right)$ and $1.84^{6} / \mathrm{mm}^{3}$ $\left(\mathrm{SEM}=4.96 \times 10^{5}\right)$, and lower in AIN and MN, $1.21 \times 10^{6}$ $\left(\mathrm{SEM}=3.04 \times 10^{5}\right)$ and $1.12 \times 10^{6} / \mathrm{mm}^{3}\left(\mathrm{SEM}=3.3 \times 10^{5}\right)$ (ANOVA of mixed effect model, $d f: 3$, Chi-square: 29.38 , $\left.p 1.87 \times 10^{-6}\right)$. The vGluT2+ bouton density differed less intensely than the vGluT1 between subnuclei, although there was still a significant difference (ANOVA of mixed effect model, $d f: 3$, Chi-square: $7.85, p 0.049$ ), mainly due to the lower density of vGluT2 in AIN. The mean density for vGluT2 was $1.7^{6} / \mathrm{mm}^{3}\left(\mathrm{SEM}=8.29 \times 10^{4}\right)$ for the total 

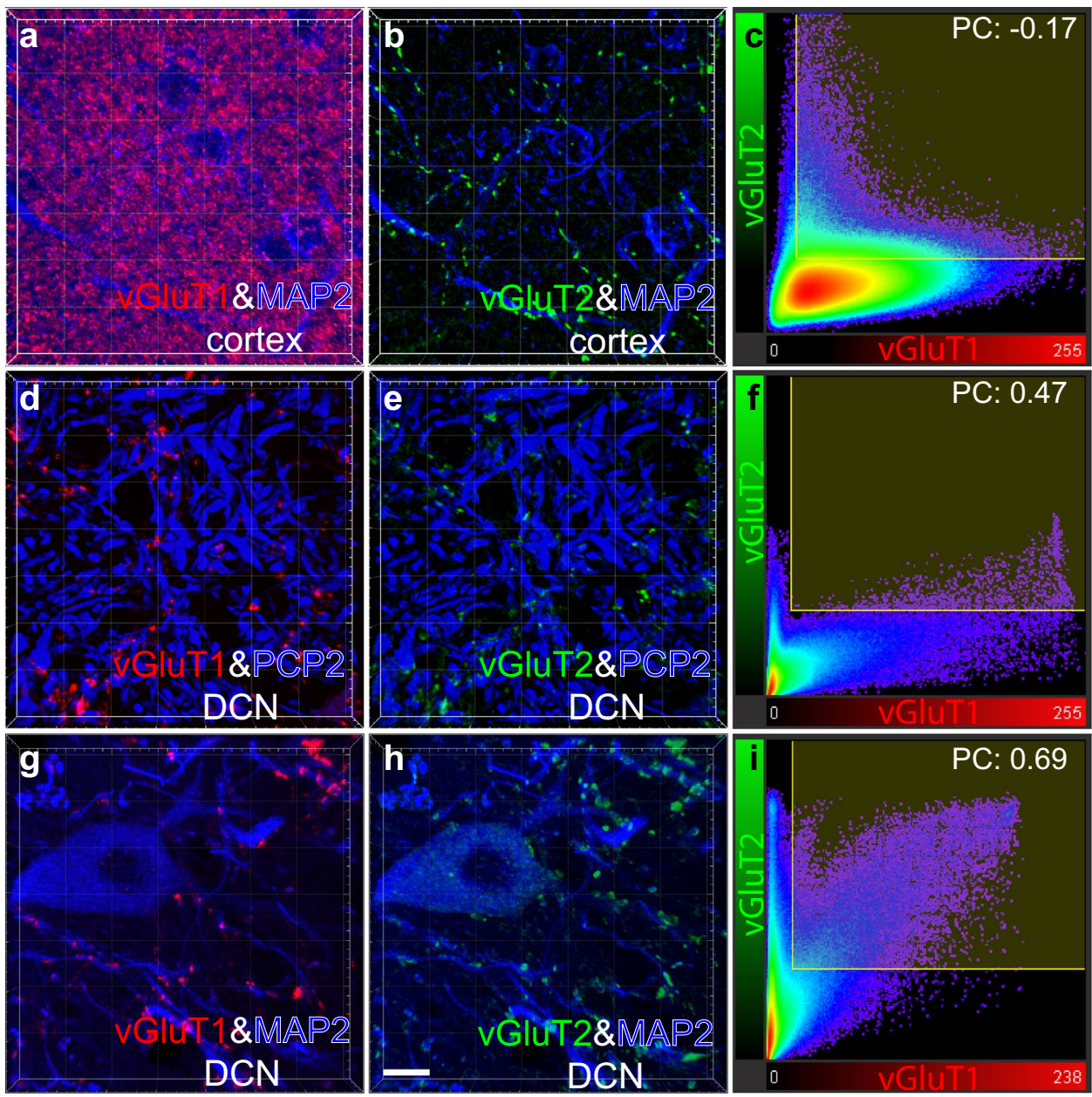

Fig. 1 vGluT1+and vGluT2+staining patterns in the cerebellum. The staining pattern of vGluT1, vGluT2 and MAP2 in the cerebellar molecular layer is shown in $\mathbf{a}, \mathbf{b}$. The parallel fibers are detected by vGluT1 antibody (red), while the climbing fiber terminals are detected by vGluT2 antibody (green). The dendrites of Purkinje cells are labelled by MAP2 (Microtubule-associated protein 2, blue) (a, b). The colocalization analysis of vGluT1 and vGluT2 is shown in c, with Pearson's coefficient (PC) -0.17 . The vGluT1, vGluT2 and PCP2 staining pattern in DCN is shown in d, e. The Purkinje cell axons are labelled by PCP 2 in blue. The excitatory presynapses are

DCN. The vGluT1 and 2+ bouton density data were power transformed to secure normal distribution and equal variance to satisfy the criterion for the statistic tests (Fig. S5a, c). The density and the volume of vGluT1+ and vGluT2+ boutons are shown for the four nuclei (Fig. 3a, b). The co-labelling of vGluT1 and vGluT2 is comparable in the AIN, PIN and $\mathrm{LN}$, around $30 \%$, with slightly lower values in $\mathrm{MN}$ (Fig. 3c). However, both the rat vGluT1 and vGluT2 colabelling, which were obtained from our previous study, are significantly lower in the rat DCN than in the macaque DCN (Fig. 3d). The mean vGluT1+ and $2+$ bouton volume is around $0.7 \mu \mathrm{m}^{3}$ and $0.9 \mu \mathrm{m}^{3}$, respectively (Fig. 3b). The vGluT1 volume was slightly larger in the $\operatorname{AIN}\left(0.78 \mu \mathrm{m}^{3}\right)$ labelled by vGluT1 (red) or vGluT2 (green) or both. The colocalization analysis of the vGluT1 and vGluT2 in DCN is shown in $\mathbf{f}$ and yields a PC of 0.47. Another example from the DCN is shown in $\mathbf{g}, \mathbf{h}$. The vGluT1, vGluT2 and MAP2 staining shows that the majority of excitatory boutons labelled by vGluT1 (red) or vGluT2 (green) contact the DCN dendrites labelled by MAP2 (blue) $(\mathbf{g}, \mathbf{h})$. The pearson's coefficient of 0.69 indicates colocalization of vGluT1 and vGluT2 in the DCN (i). Scale bar in $\mathbf{h}$ is $10 \mu \mathrm{m}$ and applies also to images (a, b, $\mathbf{d}, \mathbf{e}, \mathbf{g})$

and LN $\left(0.8 \mu \mathrm{m}^{3}\right)$ than in the other DCN (MN: 0.61 and PIN: $\left.0.70 \mu \mathrm{m}^{3}\right)$ and the vGluT2 volumes was larger in the LN $\left(0.97 \mu \mathrm{m}^{3}\right)$ than in the other DCN (PIN: 0.84, AIN: 0.89, MN: $0.86 \mu^{3}$ ), albeit with a larger variability. A one-way ANOVA was used to test the variance by different nuclei classification and the results are summarized in Table 2.

We also scrutinized our reconstructed vGluT 1 and 2 boutons for any cluster outliers, such as axonal regions where the vGluTs are detected on their way to the presynaptic terminals (Mao et al. 2018). These elongated fragments would have a large ellipticity prolate value (close to 1 ) and a small diameter. Figure 4 displays density color-coded scatter plots of vGluT1 and 2 presynapse diameter vs. the shape 

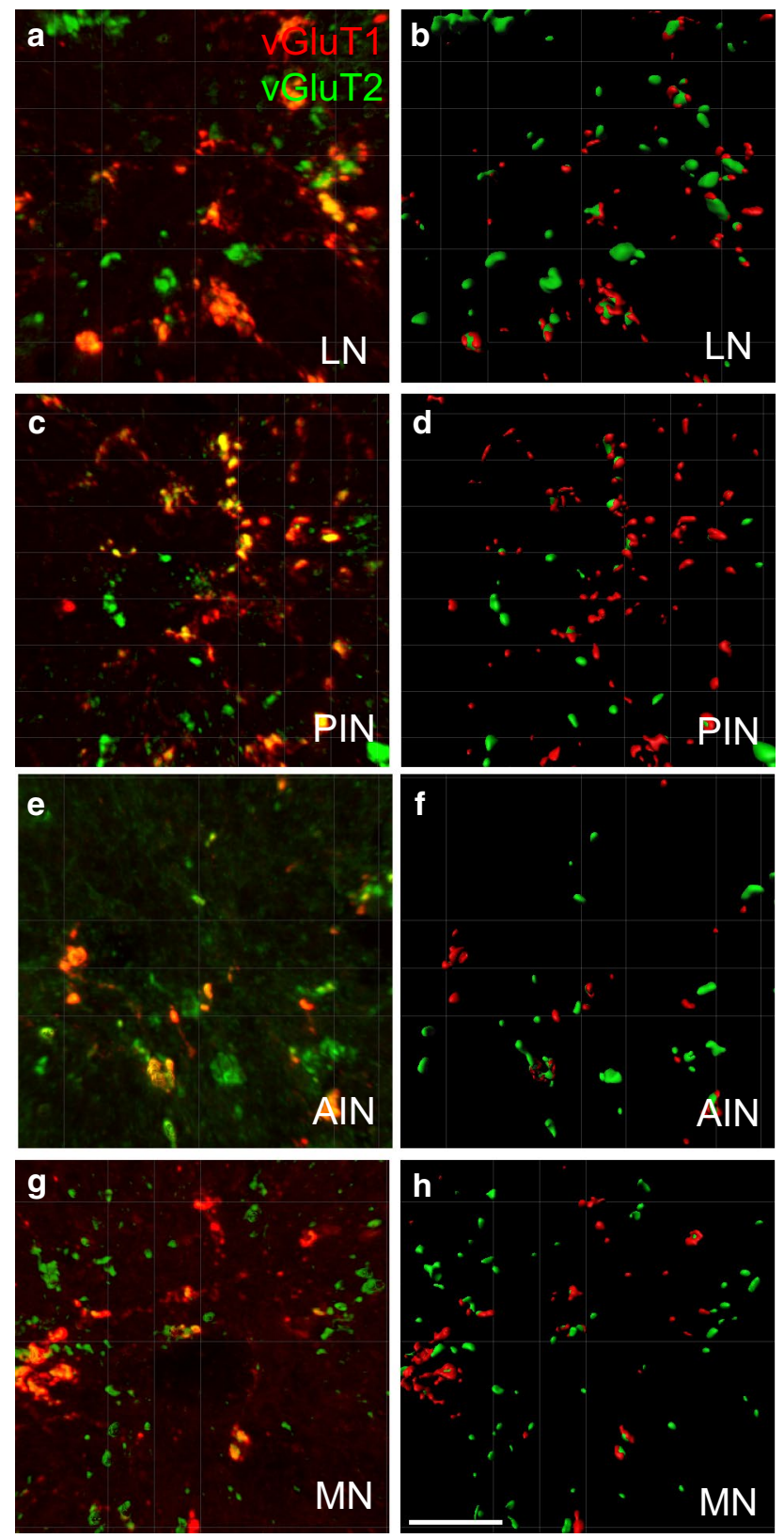

Fig. 2 Examples of vGluT1 and 2 staining in the monkey DCN. Comparison of original microscopic views $(\mathbf{a}, \mathbf{c}, \mathbf{e}, \mathbf{g})$ and surfacerendered views $(\mathbf{b}, \mathbf{d}, \mathbf{f}, \mathbf{h})$ from vGluT1 and vGluT2 stained boutons in different nuclei. The Ridler-Calvard algorithm was used for the red channel, vGluT1, while the thresholding for vGluT2 was user defined at $68 / 255$. Scale bar $10 \mu \mathrm{m}$ in $\mathbf{h}$ applies to all other images as well

parameter ellipticity prolate for the different DCN. Data show one large cluster with diameters larger than $0.5 \mu \mathrm{m}$ and ellipticity prolate larger than 0.2 and one small cluster with ellipticity prolate smaller than 0.2 . Based on these values they cannot be considered as axon fragments, because of their round shape. The number of boutons in the small cluster in the MN, AIN, PIN and LN amounted to $2.9 \%$, $3.8 \%, 4.1 \%, 3.8 \%$ for vGluT1 and $4.2 \%, 3.8 \%, 3.6 \%, 4.2 \%$ for vGluT2, respectively. These boutons may come from a distinct population, e.g., the intrinsic DCN glutamatergic synapses.

\section{Number of vGluT1+ and $2+$ bouton per neuron}

We calculated the mean bouton number per DCN neuron on the basis of our vGluT1 and vGluT2 labelled boutons in the current study and the neuron density in our previous study (Hamodeh et al. 2014, 2017). The vGluT1 and 2 bouton number per neuron is similar in rat and monkey. However, the vGluT1 bouton number per neuron is significantly lower in the macaque LN/dentate (Fig. 5a). The total excitatory synaptic number per neuron was calculated by subtracting the co-labelled vGluT1 bouton number per neuron from the total vGluT1 bouton number per neuron and adding the total vGluT2 bouton number per neuron (Fig. 5 c, d). The total bouton number per neuron in macaque DCN is lower than in the rat, except for the MN. Within the macaque, PIN has the largest bouton number per neuron (Fig. 5c, d).

\section{Discussion}

In this study, we quantified vGluT1 and 2 in a region of a primate brain for the first time by also taking the confounding contribution of lipofuscin autofluorescence into account. Our quantification shows that the largest differences are observed between the DCN in the vGluT1 densities. This result is in agreement with our previous observation finding similar differences in the rat vGluT1 densities (Mao et al. 2018). This result also confirms that our observation of variation in the dendritic length density in the macaque DCN (Hamodeh et al. 2014, 2017) is accompanied by similar vGluT1 variations, i.e., regions with higher dendritic length densities show higher vGluT1+densities. In addition, our results also point to some important differences between the rat and monkey LN/dentate, also confirming our previous dendritic quantifications showing hyposcalled dendrites in the LN/dentate. We will first discuss the potential biases and limitations of our method and then return to the interpretation of our results.

Our quantitative approach used systematic random sampling of series of multiple immunofluorescence-stained sections. In total we acquired 168 probes with 3D-stacks covering the monkey DCN from two monkeys. We adopted a new approach that involves recording an additional lipofuscin channel rather than using chemicals to diminish the lipofuscin autofluorescence. This channel was used to mask the lipofuscin fluorescence from the other fluorescence channels. Although the $405 \mathrm{~nm}$ wavelength laser used to excite the lipofuscins had the potential to bleach other fluorophores, we did not observe any indications that this was the 
Fig. 3 Boxplots of the density, volume and co-labelling of vGluT1- and vGluT2-stained boutons in different nuclei. a-c The results from the rhesus monkey DCN. a The density of vGluT1 and vGluT2 profiles. The vGluT1 density is highest in LN/dentate and PIN and lowest in MN and AIN $\left(p=1.87 \times 10^{-6}\right)$. The vGluT2 density is comparable between nuclei $(p=0.049)$ except for a lower density in the AIN. b The volumes of vGluT1 and 2 profiles. The mean vGluT1 and vGluT2 bouton volumes are $0.7 \mu \mathrm{m}^{3}$ and $0.9 \mu \mathrm{m}^{3}$, respectively. c Co-labelled percentage of vGluT1 and vGluT2 is compared in different nuclei of the monkey, with the average value of $30 \%$ for vGluT1 and $31 \%$ for vGluT2. d The co-labelling of vGluT1 and vGluT2 is significantly lower in the rat (around $15 \%$ ) than in the monkey

\section{a}

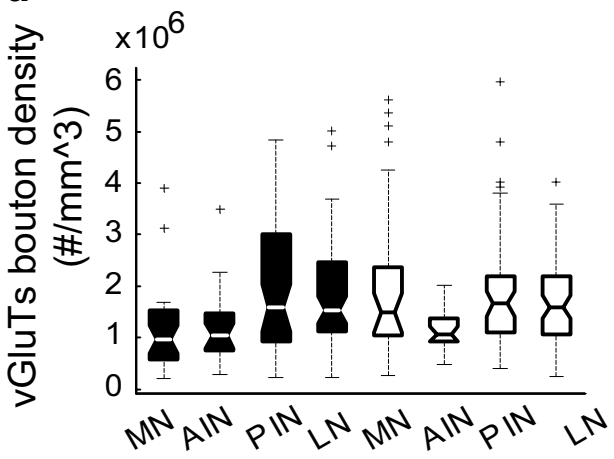

C

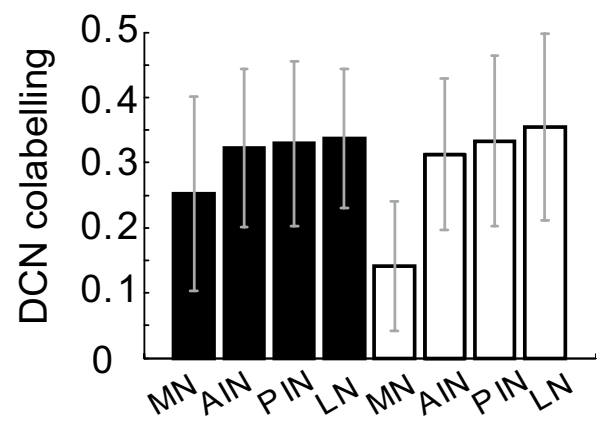

b

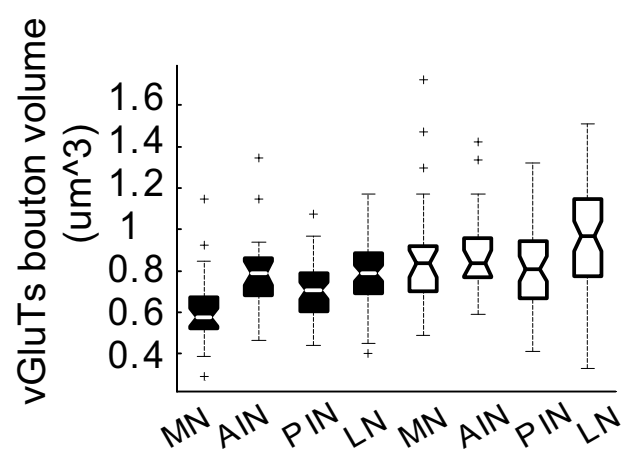

d dvGluT1 $\square$ vGluT2

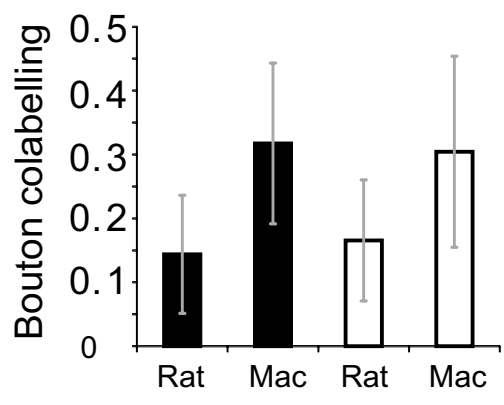

Table 2 Post hoc analysis (Tukey) of power transformed vGluT1+ and vGluT2+ bouton density and volume size

\begin{tabular}{llllllll}
\hline Threshold level & $\begin{array}{l}\text { Depend- } \\
\text { ent vari- } \\
\text { ables }\end{array}$ & $d f$ & Lambda & ICC & Chisq & $p$ value & Post hoc test \\
\hline vGluT1 & & & & & & & \\
R-C (23/255) & Density & 3 & 0.25 & 0.0526 & 29.38 & $1.87 \times 10^{-6}$ & MN and AIN $<$ PIN and LN \\
R-C (23/255) & Volume & 3 & 1 & 0.0338 & 35.69 & $8.69 \times 10^{-8}$ & MN $<$ AIN; MN $<$ PIN < LN \\
vGluT2 & & & & & & & \\
$68 / 255$ & Density & 3 & 0.05 & 0.0526 & 7.85 & 0.049 & n.s. \\
$68 / 255$ & Volume & 3 & 0.25 & 0.0284 & 9.47 & 0.023 & PIN < LN \\
\hline
\end{tabular}

The $d f$ denotes degree of freedom; lambda denotes the power used for power transformation to ensure normality and equal variance and ICC is the intracluster correlation (indicates the amount of dependence in the data). $p$ values were obtained by ANOVA. Post hoc test shows the comparisons which are statistically significant. $p$ values were adjusted by Holm-Bonferroni correction case at our laser excitation powers. We generated surfaces from the lipofuscin fluorescence and used these to mask the other channels. When the lipofuscin regions are masked, their intensities change to zero. Therefore, if the structure of interest is also located in the cytoplasm, they could be affected. In our case, this did not occur since the glutamate transporters we quantified were located in the presynapses.

One of our main finding is that the density of vGluT1labelled boutons is higher in phylogenetically newer parts of DCN, the LN/dentate and PIN, thus confirming our previous findings in the rat (Mao et al. 2018). This is also in agreement with our previous result of higher dendritic length density in these parts of the DCN. Nevertheless, the comparison of the vGluT1 densities showed that the monkey DCN $\left(1.5 \times 10^{6}\right.$ vs. $\left.6.1 \times 10^{6}\right)$ had a lower overall synaptic density of about $25 \%$ of the rat values (Mao et al. 2018). This is comparable to the reduction in neuronal density $(8640$ vs. 25,300 ) to $34 \%$ of the rat densities and shows that the overall vGluT1 synapses per neuron remain comparable. A comparison of the dendritic length density (Hamodeh et al. 2017) of monkeys vs. rats ( 51.5 vs. $\left.39.8 \mathrm{~m} / \mathrm{mm}^{3}\right)$, shows a lower reduction to $77 \%$ of the rat values and would point to a general reduction in the number of vGluT synapses per dendritic length. Excitatory synapses to the DCN neurons 

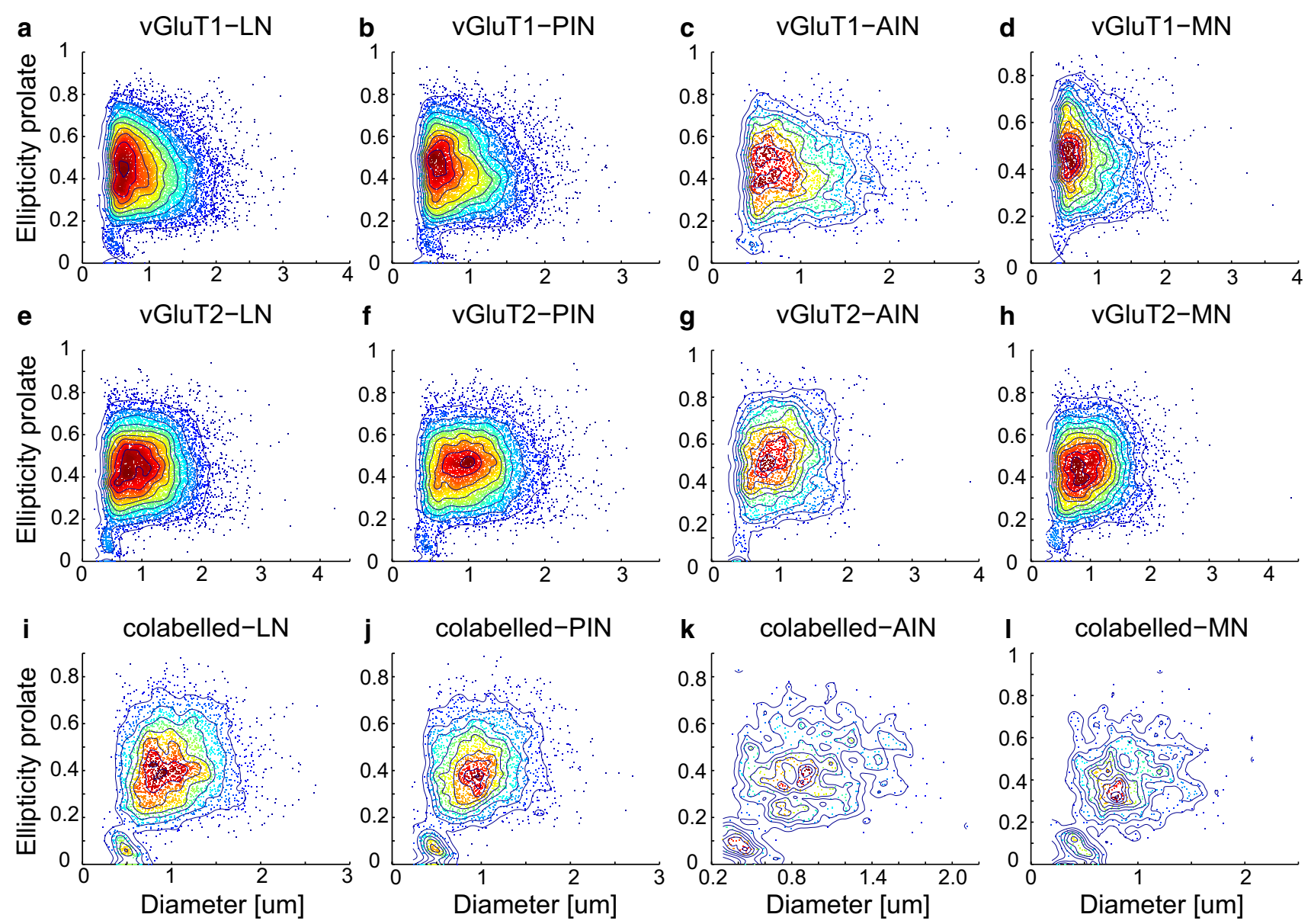

Fig. 4 Intensity color-coded scatter plots. Scatter plots of two shape parameters (ellipticity prolate and diameter) and their relation to the profile diameters for vGluT1 (a-d) and vGluT2 (e-h). Plotted are the values for the different DCN subnuclei. The overall pattern is very similar, with one large cluster encompassing most of the profiles and

one smaller cluster (to the lower left of the larger cluster) showing special subpopulations. The co-labelled boutons show similar shapes between nuclei $(\mathbf{i}-\mathbf{l})$. The color coding indicates the intensity of the scatter plot. Red represents higher and blue represents lower intensities

largely originate from the precerebellar nuclei. The largest of these nuclei is the basilar pontine nuclei which increase considerably in size and neuron number in primates. Therefore, we would have anticipated a higher number of vGluT synapses per neuron in the LN/dentate and PIN.

\section{Comparable number of excitatory synapses per neuron in rat and monkey DCN}

One explanation of the comparable values of excitatory synapses per neuron could be due to the modular organization of the cerebellum with similar number of units/neurons per module. However, other, potentially confounding factors may have led to a reduction of vGluT immunostaining in the monkey. The antibodies we used were optimized to stain the rodent's vGluT c-terminal (unspecified peptide length for vGluT1 and amino acids 510-582 for vGluT2). A comparison of the amino acid sequence of the two transporters between rat and macaca mulatta shows that vGluT1 has an essentially conserved sequence, whereas the vGluT2 shows multiple amino acid changes in the last 72 amino acids. It is, therefore, possible that the vGluT2 immunostaining in the monkey tissue could be altered, which would also explain the higher threshold used for vGluT2 compared to the rat. However, a comparison of the staining pattern within the molecular layer did not show colocalization at above-threshold intensities for the vGluT2+ only climbing fibers and the vGluT $1+$ only parallel fibers. Our calculation of the individual excitatory inputs per neuron was based on different individuals and this may have introduced additional variability in our results. We used systematic unbiased sampling in both cases and tried to minimize the influence of such bias. Finally, our two monkeys, aged 13 and 18 years, may have already shown signs of age-related synapse elimination. As anticipated, our quantification of the lipofuscin did show a higher lipofuscin density in the older monkey (Gilissen et al. 

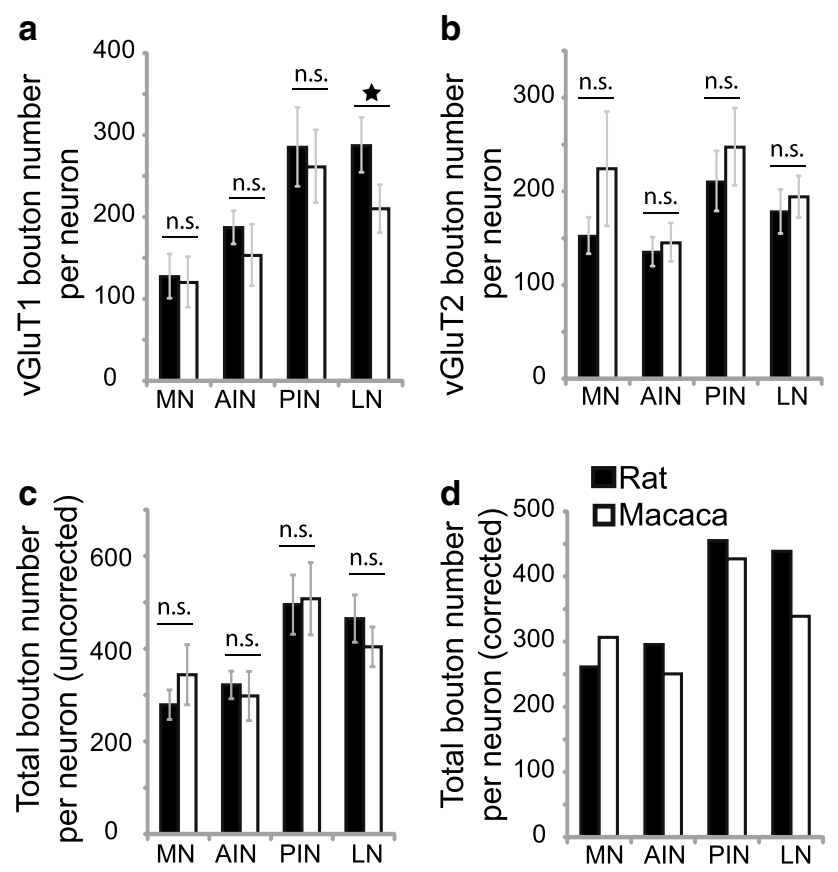

Fig. 5 Bouton number per individual DCN neuron. The vGluT1- and vGluT2-labelled boutons are normalized by the neuronal density and compared in the rat and monkey DCN. The vGluT1 bouton number per neuron is similar in the rat and monkey, except in the $\mathrm{LN}$ in which the (95\% confidence intervals) error bars do not overlap (a). The vGluT2-labelled bouton per neuron is similar in the rat and monkey DCN (b). The sum of vGluT1 and vGluT2 (uncorrected total) bouton number per neuron (c) was adjusted to remove the twice counted double-labelled boutons. This was done by subtracting the co-labelled vGluT1 bouton number per neuron from the total vGluT1 bouton number per neuron and then adding the total vGluT2 bouton number per neuron (d, "corrected"). In the monkey, the total bouton number per neuron is highest in the PIN, with a value of 427 boutons per neuron

2001). However, we did not observe systematically lower vGluT densities in the older monkey (D98 vs. H01:1.5 $\times 10^{6}$ vs. $2.3 \times 10^{6} / \mathrm{mm}^{3}$ for vGluT1 and $1.8 \times 10^{6}$ vs. $1.2 \times 10^{6} \%$ $\mathrm{mm}^{3}$ for vGluT2). In summary, our data could point to the importance of a rather constant number of excitatory synapses per DCN neuron, reflecting a limited number of input units per DCN neuron.

\section{Increased vGluT1 and 2 co-labelling in the monkey DCN}

One unexpected finding of our study is that the monkey DCN contain a higher percentage of vGluT1 and 2 colabelled presynapses than those of the rat DCN (30 vs. $15 \%)$. This is probably due to the fact that the density of vGluT2 in the monkey is higher than in the rat. The cerebellum is one of the few brain regions that shows the presence of both kinds of vGluTs (in climbing and mossy fibers), as well as presynapses with both kinds of vGluTs in a subset of mossy fibers. Developmental studies have shown that, during the early stages of development, vGluT2 synapses change to vGluT1 following the switch of vGluT2 mRNA expression to vGluT1 mRNA expression in the neuron somata (Miyazaki et al. 2003). This suggests that a change occurs at the transcription and translation level of protein synthesis. This could thus imply that the mossy fibers retain a status comparable to that of the early stages of development, allowing for prolonged modification from vGluT2 to vGluT1. A different scenario emerges if we look at a recent learning/plasticity study in the DCN. The study by Boele and colleagues showed an increase in basilar pontine nuclei vGluT2 mossy fiber presynapses in the DCN following Pavlovian eyeblink conditioning (Boele et al. 2013). So far, it remains unclear whether this kind of learning in the DCN requires the sprouting and formation of new vGluT2 synapses, or whether it is achieved by an increase in vGluT2 levels in vGluT1 mossy fiber collaterals. The latter has not been described so far and, therefore, currently appears to be the less likely scenario.

\section{Hyposcaling of vGluT1+ inputs to the primates LN/ dentate}

A further new finding of our study is that there is a lower number of vGluT1+excitatory synapses per neuron in the LN/dentate of the rhesus monkey compared to the rat's LN/ dentate. This result tallies well with the lower dendritic length per neuron that we already reported in the monkey LN/dentate (Hamodeh et al. 2017) and lends further support to our model of hyposcalled dendrites that underlie the special architecture of the LN/dentate. The hyposcaled dendritic arbors (Hamodeh et al. 2017) and vGluT1+inputs (this study) could allow for an increase in the number of independent/segregated parasagittal strips in the cerebellar hemispheres connected to the LN/dentate, which would confirm a recent prediction (Jorntell 2017). A theory proposed by van Essen (1997) on how and why structures in the central nervous system tend to fold was based on the amount of mechanical tension that arises from the connectivity pattern of a given brain region (Van Essen 1997). If we assume that the hyposcalled dendrites with smaller region of influence and with less excitatory synaptic connections would lead to less tightly connected tissue, then these less tightly connected regions would lead to a reduction of tension, which in turn would lead to some indentation and folding at these sites during the ontogenetic and phylogenetic volume increase of the nucleus. These changes could then first lead to the emergence of a torus-like or cup-shaped nucleus, as indeed the rhesus monkey LN/dentate exhibits (Sultan et al. 2010), whereas, in the nucleus with more similar connectivity tension would be basically the same all across the nucleus and would help in preserving a globose structure. 


\section{Significant statement}

We have quantified the excitatory synapses by studying the vesicular glutamate transporter (vGluT) 1 and 2 in the primate cerebellar nuclei using a novel approach to mask lipofuscin autofluorescence. Our findings confirm our previous data from the rat of a higher vGluT1+ bouton density in the phylogenetically newer cerebellar nuclei of the macaque. We also find a similar number of excitatory boutons per neuron comparing rat and monkey pointing to a conserved modular organization of the cerebellum with similar number of neurons per module. Our findings also confirm our model of hyposcalled dendrites and vGluT1+inputs to the specifically enlarged $\mathrm{LN} /$ dentate of primates.

Acknowledgements HM was funded by the Chinese Scholarship Council. This study was also supported by the Hertie Foundation, Tübingen University, the German Research Foundation (DFG SU171.3-1 grant) and (FS) the Department of Integrative medical biology, Umeå University. We are thankful to Ute Grosshennig for excellent assistance with the histology.

Author contributions $\mathrm{SH}$ sectioned the monkey cerebellum and AS assisted with the LSM multi-channel setup and lambda mode scanning. HM conducted the staining and acquired the data. HM and FS analyzed the data and prepared the manuscript. FS designed the project and was responsible for securing funding.

\section{Compliance with ethical standards}

Conflict of interest The authors declare no competing financial interests.

Ethical approval All animal experiments were carried out in accordance with the Society for Neuroscience and local German authorities (approved by the regional authorities (Regierungspräsidium Tübingen).

Open Access This article is distributed under the terms of the Creative Commons Attribution 4.0 International License (http://creativeco mmons.org/licenses/by/4.0/), which permits unrestricted use, distribution, and reproduction in any medium, provided you give appropriate credit to the original author(s) and the source, provide a link to the Creative Commons license, and indicate if changes were made.

\section{References}

Aarts E, Verhage M, Veenvliet JV, Dolan CV, van der Sluis S (2014) A solution to dependency: using multilevel analysis to accommodate nested data. Nat Neurosci 17:491-496

Billinton N, Knight AW (2001) Seeing the wood through the trees: a review of techniques for distinguishing green fluorescent protein from endogenous autofluorescence. Anal Biochem 291:175-197

Boele HJ, Koekkoek SK, De Zeeuw CI, Ruigrok TJ (2013) Axonal sprouting and formation of terminals in the adult cerebellum during associative motor learning. J Neurosci 33:17897-17907

Boulland JL, Qureshi T, Seal RP, Rafiki A, Gundersen V, Bergersen LH, Fremeau RT Jr, Edwards RH, Storm-Mathisen J, Chaudhry
FA (2004) Expression of the vesicular glutamate transporters during development indicates the widespread corelease of multiple neurotransmitters. J Comp Neurol 480:264-280

Braitenberg V, Schüz A (1998) Cortex: statistics and geometry of neuronal connectivity. Springer, Berlin

Brizzee KR, Ordy JM, Kaack B (1974) Early appearance and regional differences in intraneuronal and extraneuronal lipofuscin accumulation with age in the brain of a nonhuman primate (Macaca mulatta). J Gerontol 29:366-381

Eilers PH, Goeman JJ (2004) Enhancing scatterplots with smoothed densities. Bioinformatics 20:623-628

Fremeau RT Jr, Voglmaier S, Seal RP, Edwards RH (2004) VGLUTs define subsets of excitatory neurons and suggest novel roles for glutamate. Trends Neurosci 27:98-103

Garcia-Marin V, Ahmed TH, Afzal YC, Hawken MJ (2013) Distribution of vesicular glutamate transporter 2 (VGluT2) in the primary visual cortex of the macaque and human. J Comp Neurol 521:130-151

Gilissen EP, Dhenain M, Allman JM (2001) 27-brain aging in strepsirhine primates. In: Hof PR, Mobbs CV (eds) Functional neurobiology of aging. Academic, San Diego, pp 421-433

Haass-Koffler CL, Naeemuddin M, Bartlett SE (2012) An analytical tool that quantifies cellular morphology changes from three-dimensional fluorescence images. JoVE. https://doi. org/10.3791/4233

Hamodeh S, Sugihara I, Baizer J, Sultan F (2014) Systematic analysis of neuronal wiring of the rodent deep cerebellar nuclei reveals differences reflecting adaptations at the neuronal circuit and internuclear level. J Comp Neurol 522:2481-2497

Hamodeh S, Bozkurt A, Mao H, Sultan F (2017) Uncovering specific changes in network wiring underlying the primate cerebrotype. Brain Struct Funct 222:3255-3266

Hioki H, Fujiyama F, Taki K, Tomioka R, Furuta T, Tamamaki N, Kaneko T (2003) Differential distribution of vesicular glutamate transporters in the rat cerebellar cortex. Neuroscience 117:1-6

Hisano S, Sawada K, Kawano M, Kanemoto M, Xiong G, Mogi K, Sakata-Haga H, Takeda J, Fukui Y, Nogami H (2002) Expression of inorganic phosphate/vesicular glutamate transporters (BNPI/VGLUT1 and DNPI/VGLUT2) in the cerebellum and precerebellar nuclei of the rat, Brain research. Mol Brain Res 107:23-31

Jorntell H (2017) Cerebellar physiology: links between microcircuitry properties and sensorimotor functions. J Physiol 595:11-27

Lee KH, Mathews PJ, Reeves AM, Choe KY, Jami SA, Serrano RE, Otis TS (2015) Circuit mechanisms underlying motor memory formation in the cerebellum. Neuron 86:529-540

Mao H, Hamodeh S, Sultan F (2018) Quantitative comparison of vesicular glutamate transporters in rat deep cerebellar nuclei. Neuroscience 376:152-161

Miyazaki T, Fukaya M, Shimizu H, Watanabe M (2003) Subtype switching of vesicular glutamate transporters at parallel fibrePurkinje cell synapses in developing mouse cerebellum. Eur J Neurosci 17:2563-2572

Ridler TW, Calvard S (1978) Picture thresholding using an iterative selection method. IEEE Trans Syst Man Cybern 8:630-632

Roederer M, Murphy RF (1986) Cell-by-cell autofluorescence correction for low signal-to-noise systems: application to epidermal growth factor endocytosis by $3 \mathrm{~T} 3$ fibroblasts. Cytometry 7:558-565

Steinkamp JA, Stewart CC (1986) Dual-laser, differential fluorescence correction method for reducing cellular background autofluorescence. Cytometry 7:566-574

Sultan F, Hamodeh S, Baizer JS (2010) The human dentate nucleus: a complex shape untangled. Neuroscience 167:965-968 
Terman A, Brunk UT (2004) Lipofuscin. Int J Biochem Cell Biol 36:1400-1404

Van Essen DC (1997) A tension-based theory of morphogenesis and compact wiring in the central nervous system. Nature 385:313-318
Publisher's Note Springer Nature remains neutral with regard to jurisdictional claims in published maps and institutional affiliations. 